\title{
Desarrollo de la Teoría de la Mente en niños y niñas de Educación Infantil
}

\author{
Lucía Medina Martínez, Sandra María Rivas García, Natalia Hidalgo Ruzzante y Alfonso \\ Caracuel Romero. Universidad de Granada
}

Recepción: 29 de julio de 2016 | Revisión: 6 de septiembre de 2016 | Aceptado: 19 septiembre de 2016 Correspondencia: Natalia Hidalgo Ruzzante | Orcid ID: 0000-0002-9952-9478 | Email: nhidalgo@ugr.es Citar: Medina, L., Rivas, S., Hidalgo-Ruzzante, N. y Caracuel, A. (2016). Desarrollo de la Teoría de la Mente en niños y niñas de Educación Infantil. ReiDoCrea, 5, 185-.

\begin{abstract}
Resumen: Antecedentes: Conceptos como empatía y Teoría de la Mente (ToM) han resurgido en educación gracias a la evidencia sobre su importancia en la vida de las personas. Objetivo: Estudiar la relación entre los diferentes componentes de la ToM y determinar la relación de variables sociodemográficas y educativas en el desarrollo de la ToM en alumnado de Educación Infantil. Método: 25 niños/as con edades comprendidas entre 5 y 6 años fueron evaluados. Se empleó una escala validada para evaluar ToM (EToM). Los ítems consisten en seis situaciones conflictivas como diversidad de creencias, deseos, emociones o conocimientos, presentados utilizando personajes y materiales lúdicos. Resultados: Los ítems de la escala, correspondientes a diferentes componentes de la ToM, están relacionados entre ellos con los adyacentes, no con los más distantes en el orden teórico establecido en la escala. No se han obtenido resultados significativos que indiquen la relación de edad, sexo, dificultades del lenguaje y tener hermanos/as con el desarrollo de la ToM. Conclusiones: EToM contiene ítems que pueden ser considerados indicadores del desarrollo evolutivo de la ToM en preescolares. Investigaciones con muestras más amplias podrán determinar la relación de la ToM con el sexo, la edad, el lenguaje y la convivencia con hermanos.
\end{abstract}

Palabras clave: Primera infancia | Desarrollo emocional

Development of the Theory of Mind in children of Pre-elementary education

\begin{abstract}
Background: In recent years the concepts of empathy and Theory of Mind (ToM) have reemerged in the area of education, due to evidence of their value in people's lives. Objective: To study what relation exists between the components of ToM, and determine the relationship of sociodemographic and educational variables in the development of ToM in children of Pre-elementary education. Method: 25 children between 5 and 6 years old were evaluated; a validated scale to assess ToM in children was used. The items consist of six conflict situations about differences in beliefs, desires and emotions among others, presented to the students using characters and play-based materials. Results: The items of the scale, corresponding to different components of ToM are related to each other but only with adjacent items, not with the more distant ones in the theoretical order established in the scale. No significant results were obtained indicating the relationship of between age, sex, language problems and having siblings in the development of ToM. Conclusion: The ToM Scale contains items that can be considered as indicators of an evolutionary development of ToM in children in pre-elementary Education. Studies with broader samples are needed to determine the relationship between ToM and sex, age, language and living with siblings.
\end{abstract}

Key words: Early Childhood | Emotional Development

\section{Introducción}

La complejidad en los sentimientos, el razonamiento y la empatía son algunas de las características que diferencian al ser humano del resto de los seres vivos. En el contexto social es necesario intuir la conducta de las otras personas, sus conocimientos, sus intenciones y sus emociones. Esta habilidad, definida inicialmente por Premack y Woodruff (1978) como Teoría de la Mente (ToM), se corresponde con la capacidad de atribuir pensamientos e intenciones a otras personas (Aldana, 2011). Implica verse a uno mismo y a otros en términos de estados mentales (emociones y creencias), que dan como resultado el comportamiento (Wellman, Cross y Watson, 2001).

El término ToM surgió tras un estudio para determinar la capacidad de los chimpancés de reconocer los estados mentales de otros seres vivos y actuar en consecuencia (Premack y Woodruff, 1978). En primer lugar, mostraron vídeos a un chimpancé sobre una persona afrontando varios problemas. A continuación, debía elegir entre dos imágenes, una mostrando una acción que resolvía el problema y otra que no lo 
solucionaba. La elección correcta demostraría que los chimpancés son capaces de reconocer los estados mentales de otros individuos (darse cuenta de que la persona quiere solucionar el problema) y actuar en consecuencia (elegir la opción que soluciona dicho problema). De las veinticuatro situaciones, resolvió correctamente veintiuna (Premack y Woodruff, 1978).

Dennett (1989) realizó un estudio para determinar si la complejidad de la ToM en los seres humanos era igual que en los chimpancés. A pesar de que sí comprendían los deseos y creencias de otros seres vivos, eran incapaces de intuir el sentimiento que sus acciones generaban en los otros, concluyendo que sus acciones no pueden estar determinadas por ese factor.

El desarrollo de la ToM se produce a partir de los 3 años y medio (Perinat, 2007), cuando se tiene adquirida la capacidad simbólica. La importancia de un desarrollo inadecuado de la ToM radica en sus consecuencias negativas para las relaciones, con repercusión en la evolución psicológica y cognitiva del individuo. Por ejemplo, las personas con autismo o síndrome de Asperger suelen presentan dificultades para intuir lo que los demás están pensando en base a su comportamiento (Valdez, 2001) y para reconocer las creencias erróneas en los demás (Whiten, 2005), frecuentemente asociado con dificultades en las relaciones sociales.

Varios estudios con muestras infantiles han encontrado que el desarrollo de la ToM es progresivo y jerárquico, de forma que una vez adquirido un nivel, con una gran probabilidad se habrán superado los niveles anteriores de desarrollo. Este tipo de hallazgos ha sido posible gracias a la validación de la Escala de la ToM para la infancia, que reúne una serie de ítems que evalúan componentes como deseos, creencias, emociones, etc. (Wellman y Liu, 2004).

Se ha encontrado una serie de factores que pueden influir en el desarrollo de la ToM. Uno de los factores podría ser la cultura en la que se desarrolla el niño o niña. Se han comparado niños y niñas de China con los de Estados Unidos y Australia en cuanto al desarrollo de la ToM, encontrándose diferencias en el orden en el que los/as niños/as adquirieron varios de los componentes dependiendo de la cultura de origen (Wellman, Fang, Liu, Zhu y Liu, 2006).

El desarrollo de las competencias lingüísticas también podría guardar relación con la adquisición de la ToM. Conforme aumenta el léxico y la complejidad sintáctica, el desarrollo de la ToM es más rápido y eficiente, probablemente porque se comprenden de forma más adecuada las acciones tanto ajenas como propias (Resches, Serrat, Rostan y Esteban, 2010). La importancia que tiene la complejidad del lenguaje en la ToMse refleja en los hallazgos de un estudio con niños y niñas sordas de padres oyentes que presentaban retrasos sustanciales en la adquisición de la ToM, frente a hijos e hijas con sordera nacidos de padres sordos, que no mostraban retraso probablemente por la mayor riqueza comunicativa entre ambos cuando ambos han desarrollado el lenguaje de signos desde su infancia (Wellman, Fang, y Peterson, 2011). Muchas de las personas con autismo presentan déficits en las competencias lingüísticas (Resches y cols., 2010), pudiendo también contribuir a sus dificultades en la creación de una teoría de la mente de los demás.

Respecto a la posibilidad de asociaciones entre la ToM y el nivel socioeconómico de la familia, un estudio que comparó niños/as de diferentes clases sociales y económicas, concluyó que este factor no tenía repercusión en el desarrollo de esta capacidad (Shahaeian, 2015). Por otro lado, varios estudios concluyen que tener hermanos/as 
mayores influye en el desarrollo de la ToM (McAlister y Peterson, 2007). Shahaeian (2015) determinó que lo que realmente influye en el desarrollo de la ToM es el grado de interrelación con niños/as de edad similar. Por lo que la influencia de tener hermanos/as depende de la interacción que exista entre ellos. Además, se demostró que la influencia afecta tanto para los hermanos/as mayores como para los menores, es decir, ambos se benefician de la interacción (Prime, Plamondon, Pauker, Perlman y Jenkins, 2016).

Considerando que el desarrollo de la ToM puede estar influido por determinadas variables sociodemográficas y educativas, los objetivos del estudio fueron conocer la relación que guardan entre sí algunos de los componentes de la Teoría de la Mente (ToM), y determinar la relación de la edad, el sexo, dificultades de lenguaje y tener hermanos mayores con el desarrollo de la ToM en el alumnado de Educación Infantil con edades comprendidas entre 5-6 años.

\section{Método}

\section{Participantes}

La muestra estaba compuesta por los 25 alumnos y alumnas (11 niños y 14 niñas) de una clase $3^{\circ}$ de Educación Infantil de un centro educativo concertado de Granada. Las edades estaban comprendidas entre los 5 y los 6 años (media=5,6 años, desviación estándar $=0,33$ y rango=5,2-6,1). El nivel socioeconómico medio de los alumnos y alumnas del centro es medio-bajo, aunque también se encuentra alumnado con un nivel socioeconómico muy bajo.

\section{Instrumento}

Se ha adaptado la Escala de la Teoría de la Mente (EToM) utilizada en los estudios de Wellmann y Lui (2004) con materiales para niños españoles. La escala evalúa desde los componentes de nivel más básico de desarrollo de la ToM a otros más complejos y difíciles de adquirir para los niños y niñas de 5-6 años. A continuación se describen las tareas de evaluación siguiendo el orden de presentación a los participantes. El orden está establecido por el grado de complejidad o dificultad de adquisición de las mismas establecido a priori por los autores de la escala, desde el más fácil de adquirir al más difícil. Se han utilizado solo los seis primeros ítems, evitando el uso del séptimo y último por su alta complejidad para niños de 5-6 años.

Ítem 1: Deseos diversos

Objetivo: evaluar si el participante comprende que dos personas (el participante y otra persona adulta) tienen deseos diferentes sobre el mismo objeto.

Materiales: dibujos de una zanahoria y una barrita de chocolate en un papel, y una figura representativa de un adulto, el Sr. Juan.

Administración: el niño o niña ve la figura de un adulto y una hoja de papel con una zanahoria y una galleta dibujada. Le decimos "este es el señor Juan, es la hora de la merienda y quiere comer algo. Para merendar tenemos dos cosas, una zanahoria y una galleta, ¿a ti qué te gusta más?". Esta es la pregunta sobre el deseo propio (owndesirequestion). 
Si el participante elige la zanahoria se le dice: "Bien, buena elección, pero al señor Jon realmente le gustan las galletas, a él no le gustan las zanahorias" (en caso de que haya elegido la galleta, se le dice que al señor Juan le gustan las zanahorias). A continuación, se le pregunta al niño o niña la siguiente pregunta objetivo (targetquestion): "Es hora de comerse algo y el señor Juan solo puede elegir uno de los alimentos, solo uno, ¿qué crees que elegirá?, ¿una zanahoria o una galleta?"

Corrección: para que la respuesta sea correcta e indicativa de que el niño-a tiene adquirido el componente de que las personas pueden tener gustos diferentes a los suyos, debe de contestar la respuesta objetivo con la respuesta contraria a la pregunta sobre su propio deseo.

\section{Ítem 2: Diversas creencias}

Objetivo: evaluar si el participante comprende que dos personas (el participante y una niña) tienen diferentes creencias sobre el mismo objeto, cuando el niño o niña no sabe cuál es la respuesta verdadera o falsa.

Materiales: una caja de cartón, un pompón de lana con forma de arbusto y una muñeca.

Administración: el alumnado ve una figura de juguete representativa de una niña, un arbusto y una caja de cartón. Les decimos "esta es Linda, necesita encontrar a su gato". "El gato podría estar escondido en este arbusto o podría estar en la caja de cartón”. ¿Dónde crees que está en gato, en el arbusto o en la caja? (pregunta sobre la creencia propia). Si el niño-a elige el arbusto, se le responde: "Buena elección, pero Linda cree que está en la caja", "ella cree que está en la caja" (en el caso de que el niño-a diga que piensa que el gato está en la caja, se le dice que Linda cree que su gato está en el arbusto). Después se le hace la pregunta objetivo. Por lo tanto, ¿dónde buscará Linda a su gato, en el arbusto o en la caja?

Corrección: para que la tarea sea correcta el participante debe contestar a la pregunta objetivo lo contrario que a la pregunta sobre su propia creencia.

\section{Ítem 3: Acceso al conocimiento}

Objetivo: evaluar si el participante comprende la falta de conocimiento de otra persona sobre el contenido de un recipiente cuando el niño-a sabe lo que contiene.

Materiales: una caja de cartón, un perro de juguete y una muñeca.

Administración: el/la niño/a ve una caja opaca que tiene un perrito de juguete pero que no puede ser visto. Le decimos "esto es una caja, ¿qué crees que puede haber dentro?. El participante dirá cualquier cosa o que no sabe lo que hay en el interior de la caja. A continuación se abre la caja y se le muestra el contenido: "Vamos a ver, ¡hay un perrito dentro!'. Se cierra la caja y se le pregunta: "Vale, ¿qué hay en la caja?".

A continuación se acerca una figura de una chica de juguete: "Patri nunca ha visto lo que hay en el interior de la caja. Ahora llega Patri. Por tanto, ¿sabe Patri lo que hay dentro de la caja? (pregunta objetivo). ¿Ha visto Patri el interior de la caja? (pregunta de memoria). 
Corrección: para que la respuesta al ítem sea correcta, las respuestas tanto a la pregunta objetivo como a la pregunta memoria debe ser no.

\section{Ítem 4: Falsa creencia de contenido}

Objetivo: evaluar si el participante comprende el error en la creencia de otra persona sobre el contenido no esperable de un recipiente muy característico cuando el/la niño/a conoce lo que se ha introducido en el recipiente.

Materiales: una hucha en forma de cerdito, cuatro canicas, cuatro monedas y la presencia de una mujer.

Administración: el /la niño/a ve claramente una hucha con forma de cerdito. Se mueve para que suene el contenido interior y se le dice: "Esto es una hucha, ¿qué crees que hay dentro?". A continuación, la abrimos y decimos "Vamos a ver, jen realidad lo que hay dentro son canicas!'. Se cierra la hucha y se le pregunta: "Vale, ¿qué hay en la hucha?.

A continuación se acerca una figura de un chico de juguete: "Pedro nunca ha visto lo que hay en el interior de la hucha. Ahora llega Pedro. Por tanto, ¿qué cree Pedro que hay dentro de la hucha, monedas o canicas? (pregunta objetivo). ¿Ha visto Pedro en interior de la hucha? (pregunta de memoria).

Corrección: para que la respuesta al ítem sea correcta, la respuesta a la pregunta objetivo debe ser "monedas" y la respuesta de la cuestión de memoria debe de ser "no"

\section{Ítem 5: Falsas creencias explícitas}

Objetivo: evaluar si el participante comprende que la creencia de otra persona sobre el lugar en el que se encuentra un objeto debe ser errónea cuando se ha manipulado su localización mientras no estaba presente.

Materiales: dos muñecas de trapo, dos cajas de maderas y una canica.

Administración: el niño o niña ve las figuras de dos chicas de juguete, dos cajas y una bola. Se les presentan "estas son dos niñas que están jugando, Sally y Anne". A continuación se escenifica y se le cuenta: "Ellas tienen dos cajas diferentes". Estando las dos muñecas presentes se introduce la canica en una de las cajas. Después sse dice: "Anne se va" y en ese momento una de las muñecas desaparece. "Sally coge la canica y la introduce en la otra caja". Vuelve a aparecer Anne y se le pregunta: ¿Dónde pensará Anne que se encuentra la canica en este momento?

Corrección: para que la respuesta sea correcta, el participante debe responder o señalar la caja en la que se encontraba la canica en el momento que Anne y Sally estaban presentes.

\section{Ítem 6: Creencias emocionales}

Objetivo: evaluar si el participante comprende que las emociones derivadas de las creencias y los cambios en la emoción al contrastar con la realidad. 
Materiales: una rana de peluche, una caja de cartón y piedras.

Administración: el/la niño/a ve a una figura de una rana de peluche y una caja con un dibujo de cerezas. "Esto es una caja con la comida favorita (cerezas) de Rita la rana. ¿Qué crees que hay dentro de la caja?”. Entonces, el adulto pone voz a Rita, la rana y dice " $\mathrm{OOh}$ ! bien porque a mí me encantan las cerezas, son mi merienda favorita. Ahora me voy a jugar". Rita la rana desaparece de la escena.

Posteriormente la caja de cerezas se abre y el contenido se le muestra al participante, "veamos que contiene, jhay realmente piedras y no cerezas! No hay otra cosa que no sean piedras, no hay cerezas". Se cierra la caja. "¿Cuál es la merienda favorita de Rita la rana? (cerezas). Rita nunca ha visto el interior de la caja".

La rana vuelve a la escena. "Es la hora de la merienda de Rita, démosle la caja a Rita. ¿Cómo se siente Rita cuando consigue la caja triste o feliz?" (pregunta objetivo). El adulto abre la caja y deja que la rana mire dentro "¿Cómo se siente Rita después de mirar en el interior de la caja, contenta o triste?" (pregunta control sobre las emociones).

Corrección: para que la respuesta sea correcta debe responder "feliz" a la pregunta objetivo y "triste" a la pregunta control sobre las emociones.

\section{Procedimiento}

La prueba se realizó en el aula ordinaria, en una de las esquinas de la asamblea del aula, en la zona más tranquila y aislada. La prueba se llevó a cabo de uno en uno, por orden de lista de clase, mientras que sus compañeros y compañeras realizaban las tareas diarias, manteniéndose un ambiente tranquilo y lúdico. El/la niño/a que realizaba la prueba quedaba de espaldas a sus compañeros y compañeras, para impedir distracciones.

Las variables independientes del estudio fueron edad, sexo y presencia de hermanos/as mayores y la dificultad en el lenguaje evaluado por la maestra del aula. Las variables dependientes fueron los resultados en cada uno de los seis ítems de la EToM y la suma de los ítems.

\section{Análisis de datos}

Se llevaron a cabo análisis descriptivos de la muestra. Se calculó el índice Rho de Sperman para determinar la correlación entre los resultados de cada una de las 6 pruebas y el coeficiente de correlación de Pearson para la relación entre la edad y la suma de los ítems de la EToM. Se utilizó el estadístico U Mann Whitney para determinar las diferencias entre grupos de sexo, lenguaje y presencia de hermanos. Los análisis de realizaron con el programa SPSS-23.

\section{Resultados}

Para llevar a cabo el primer objetivo (conocer la relación que guardan entre sí algunos de los componentes de la ToM) se han calculado los índices de correlación de Sperman para determinar la asociación entre los resultados de cada una de las 6 pruebas. En la tabla 1 se observan correlaciones significativas entre las pruebas adyacentes, excepto entre la prueba de nivel 1 y la de nivel 2. Las correlaciones entre pruebas no adyacentes no es significativa (ver flechas interrumpidas en figura 1). 
Según los resultados, excepto entre las pruebas 1 y 2 , todas las demás se correlacionan de forma que se establece un patrón de asociaciones entre cada una y su inmediata superior e inferior en la escala de niveles de dificultad indicada en la literatura.

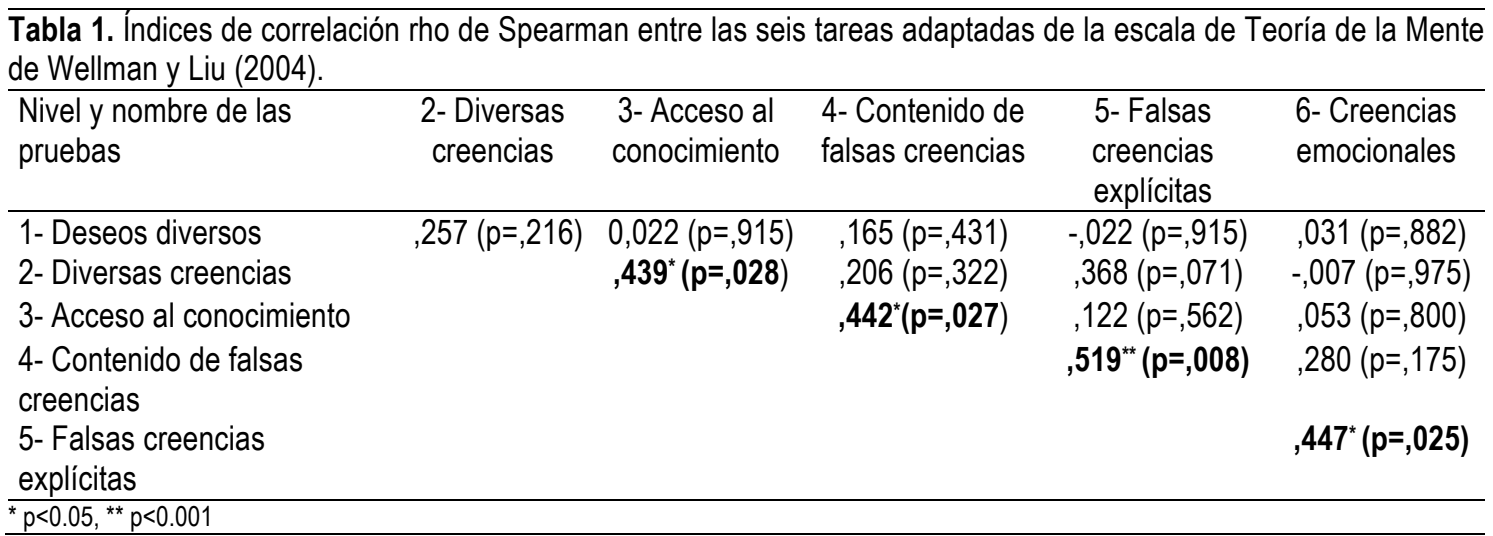

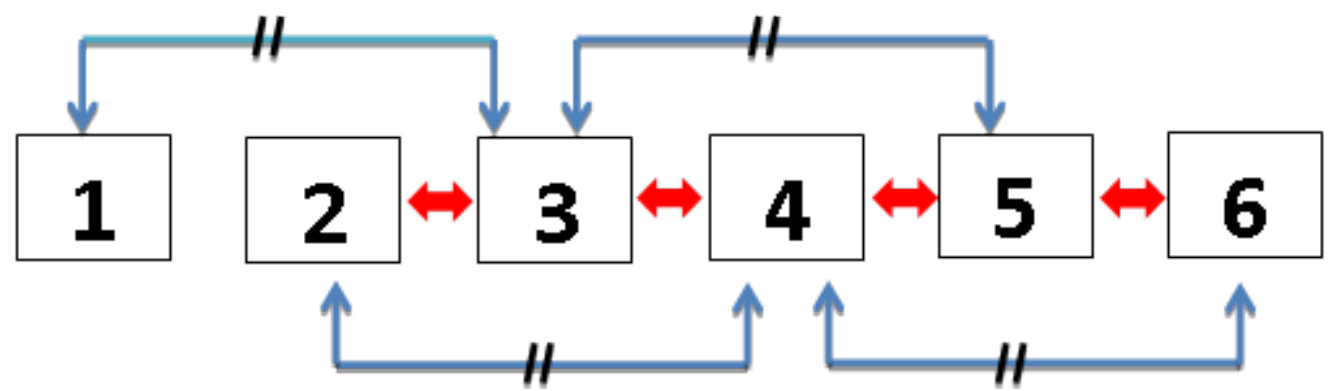

Figura 1. Representación de las asociaciones significativas (flecha roja) y no significativas (flechas azules) entre las seis pruebas.

Los porcentajes de acierto de cada uno de los hitos se pueden ver en el gráfico 1.

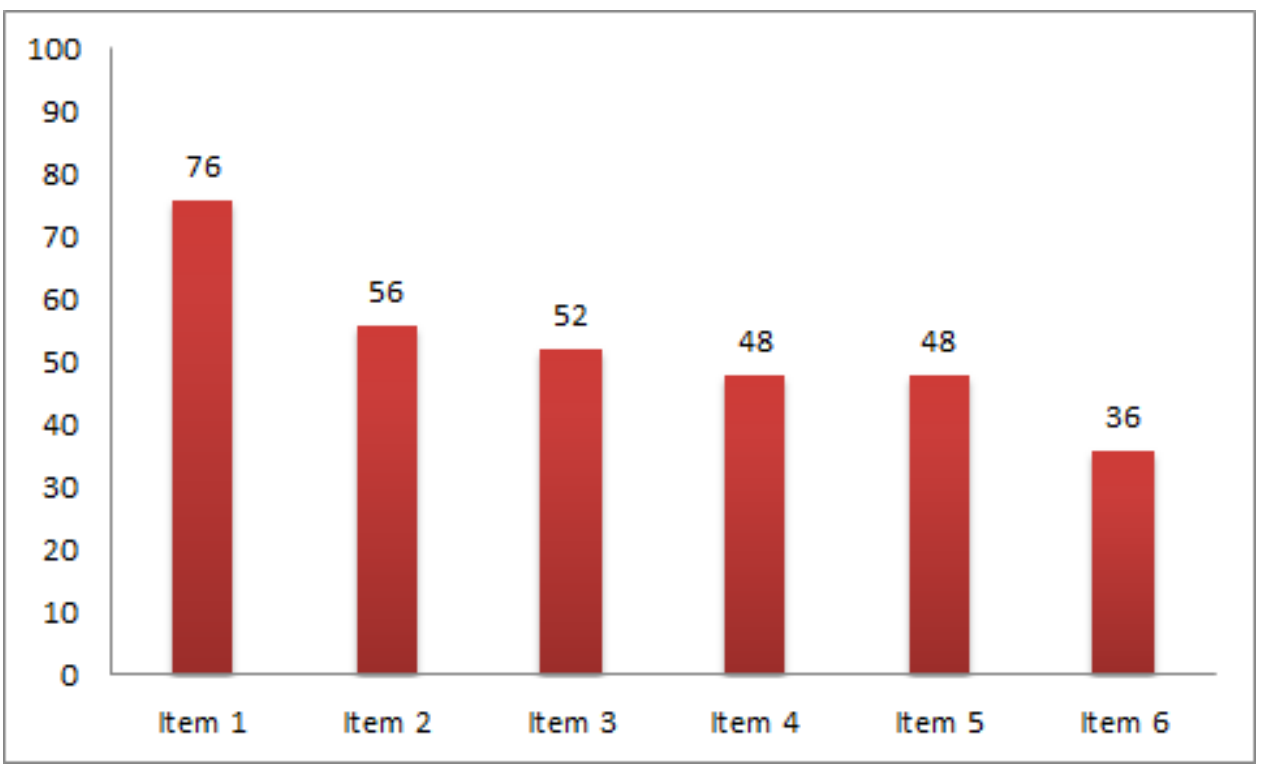

Gráfico 1. Porcentaje de acierto de cada uno de los hitos de la batería de evaluación de la ToM.

Para llevar a cabo el segundo objetivo (determinar la relación de la ToM con la edad, el sexo, dificultades de lenguaje y tener hermanos mayores) se ha realizado un 
análisis de la correlación entre la suma de los resultados de las pruebas de cada participante y su edad. No se ha encontrado una asociación significativa $(r=.085$, $p=.686$ ). Para determinar la influencia del sexo, la presencia de problemas de lenguaje y la ausencia de hermanos mayores se han comparado los correspondientes grupos y no se han obtenido diferencias significativas (Ver tabla 2).

\begin{tabular}{lcc}
\hline Tabla 2. Diferencias entre grupos de sexo, problemas de lenguaje y presencia de hermanos mayores \\
\hline Variable de agrupación & U Mann Whitney & $p$ \\
\hline Sexo & 68,5 &, 714 \\
Problemas de lenguaje & 27 &, 113 \\
Presencia de hermanos mayores & 75 &, 868 \\
\hline
\end{tabular}

\section{Discusión}

Los objetivos del estudio fueron conocer la relación que guardan entre sí algunos de los componentes de la Teoría de la Mente (ToM), y determinar la relación de la ToM con la edad, el sexo, dificultades de lenguaje y tener hermanos mayores en el alumnado de 5-6 años.

Los hallazgos indican que solo existen relaciones entre los ítems adyacentes, y no entre los que se encuentran alejados en el orden establecido a priori por los autores de la escala (Wellman y Liu, 2004). Estos resultados apoyan los hallazgos de los estudios que indican que los distintos ítems pueden ser considerados como hitos evolutivos que se adquieren progresivamente y que forman una jerárquica. La jerarquía indica que aquellos alumnos o alumnas que superen un ítem determinado, por ejemplo el cuarto, tienen una probabilidad alta de haber superado los tres anteriores, y que la probabilidad de haber adquirido el quinto y sexto es menor.

Los hallazgos encontrados indican que la edad no guarda relación con el nivel de aciertos alcanzado. Este resultado resulta contradictorio con la idea anterior de desarrollo evolutivo. Atribuimos la falta de asociación al rango escaso de edad de los participantes, todos con edades muy similares. Aunque el desarrollo evolutivo se asocia con la edad (Waber et al., 2007), el desarrollo evolutivo en el área específica de la ToM podría estar influido por otros factores, no solo por la edad.

En lo relativo al lenguaje, no se han encontrado diferencias significativas entre niños/as con dificultades de lenguaje y los que no los tienen. Sin embargo, la literatura indica que el desarrollo del lenguaje y la complejidad comunicativa influyen en el desarrollo de la ToM (Resches y cols., 2010). La falta de resultados positivos puede estar muy influida por el tamaño pequeño de la muestra y, sobre todo del grupo de niños/as con dificultades del lenguaje (el $20 \%$ del total). Otra de las explicaciones de la falta de resultados positivos puede atribuirse al método empleado para determinar la presencia de los problemas de lenguaje. La maestra fue la que indicó qué alumnos/as tenían dificultades o retrasos del lenguaje, en base al criterio de su experiencia y no mediante pruebas objetivas. Estas estimaciones sobre los participantes no son datos específicos sobre los déficits que puedan presentar los niños y niñas. Para futuros estudios es aconsejable contar con más información y basada también en el diagnóstico y consideración de especialistas del lenguaje.

Además, algunos autores apoyan que el desarrollo de la ToM se inicia desde los primeros meses de vida (antes de la adquisición del lenguaje), en los que el bebé imita 
los gestos de su madre, padre y cuidadores/as (López, Etxebarría, Fuentes y Ortiz, 2001). Realmente los gestos forman parte de esa serie de comportamientos sociales que se encuentran en la base del desarrollo de la empatía. Además de analizar el lenguaje, el estudio de estas variables previas a la adquisición del mismo podría resultar de importancia crucial en el estudio del desarrollo de la ToM.

Tampoco se han encontrado diferencias en el desarrollo de la ToM asociadas al sexo y presencia de hermanos/as. Estos resultados difieren con lo expuesto por otros autores (Wellman y Liu, 2004). Nuevamente, debemos pensar que el tamaño muestral puede ser la causa de la falta de resultados positivos.

Entre las limitaciones del presente estudio se encuentra el reducido tamaño muestral, que limita el poder estadístico de los resultados y su posibilidad de generalización. Además, algunas variables, como las dificultades del lenguaje, podrían haber sido evaluadas de un modo objetivo. En futuras investigaciones debería aumentarse el número de participantes y realizar una evaluación más precisa de las variables de estudio.

Para finalizar, debemos considerar la importancia de las investigaciones sobre el desarrollo de la ToM. Los miembros de la especie humana, al contrario que otras, nacemos con pocas capacidades desarrolladas, lo que constituye una necesidad y oportunidad para trabajarlas y fomentarlas en un proceso de enseñanza que incluya no solo aspectos cognitivos, sino también emocionales y sociales. Hoy día, en el proceso enseñanza-aprendizaje no solo las "materias básicas" deberían de ser esenciales, el aprendizaje de valores y emociones deben adquirir un papel central en la educación, especialmente por su influencia en el desempeño escolar de los niños y niñas en las tareas académicas (lectura, escritura, matemáticas, etc.) y en las estrategias que emplean para lograr éxito académico (Wellman, 2016).

Así, uno de los aspectos fundamentales para evaluar y trabajar desde la primera infancia es el desarrollo de la ToM. La evaluación del grado de adquisición de la misma y las diferentes variables que pueden influir en su desarrollo permitirá determinar si los niños y niñas están adquiriendo el nivel de desarrollo esperable y deseable que facilitará el éxito escolar, pero también la integración social y calidad de vida de los mismos.

En conclusión, la Escala de la ToM contiene ítems que pueden ser considerados como indicadores de un desarrollo evolutivo de la ToM en los niños y niñas de Educación Infantil. Sin embargo, para determinar de forma adecuada la relación de la ToM con el sexo, la edad, el lenguaje y la convivencia con hermanos/as en población española se necesitan estudios con muestras más amplias.

Referencias

Aldana, M. (2011). Teoría de la mente. Revista Digital Enfoques Educativos, 74, 4-20.

Baron-Cohen, S. (2012). Empatía cero. Nueva teoría de la crueldad. Madrid: Alianza.

Dennett, D. (1989). Condiciones de la cualidad de persona. México: Universidad Nacional Autónoma de México.

López F., Etxebarria, I., Fuentes, MJ. y Ortiz, MJ. (Eds.). (2001). Desarrollo afectivo y social. Madrid: Pirámide. 
Premack, D., \& Woodruff, G. (1978). Does the chimpanzee have a theory of mind? Behavioral and brain sciences, 1(04), 515-526.

Prime, H., Plamondon, A., Pauker, S., Perlman, M., \& Jenkins, J. (2016). Sibling cognitive sensitivity as a moderator of the relationship between sibship size and children's theory of mind: A longitudinal analysis. Cognitive Development 39, 93-102.

Resches, M., Serrat, E., Rostan, C. y Esteban, M. (2010).Lenguaje y Teoría de la Mente: una aproximación multidimensional. Infancia y Aprendizaje, 33(3), 315-333.

Shahaeian, A. (2015). Sibling, family, and social influences on children's Theory of Mind understanding: New evidence from diverse intracultural samples. Journal of Cross-Cultural Psychology, 1-16.

Valdez, D. (2001). Teoría de la Mente y espectro autista. Autismo: enfoques actuales para padres y profesionales de la salud y la educación. Buenos Aires, Argentina: Fundec.

Vergara, M.A. (s.f.). Manual de Pediatría. Recuperado 25 de julio de 2016, de http://escuela.med.puc.cl/paginas/publicaciones/manualped/DessPsicPreesc.HTML

Waber, D., De Moor, C., Forbes, P.W., Almli, C.R., Botteron, K.N., Leonard, G., Milovan, D., Paus, T., \& Rumsey, D. (2007). The NIH MRI study of normal brain development: Performance of a population based sample of healthy children aged 6 to 18 years on a neuropsychological battery. Journal of the International Neuropsychological Society, 13, 729-746.

Wellman, H.M. (2016). Cognición social y educación: teoría de la mente Social Cognition and Education: Theory of Mind. Pensamiento Educativo. Revista de Investigación Educacional Latinoamericana, 53(1), 1-23.

Wellman, H.M., \& Liu, D. (2004). Scaling of theory-of-mind tasks. Child Development, 75(2), 523-541.

Wellman, H.M., Fang, F., \& Peterson, C.C. (2011). Sequential progressions in a theory-of-mind scale: Longitudinal perspectives. Child Development, 82(3), 780-792.

Wellman, H.M., Fang, F., Liu, D., Zhu, L., \& Liu, G. (2006).Scaling of theory-of-mind understandings in Chinese children. Psychological Science, 17(12), 1075-1081.

Wellman, H., Cross, D., \& Watson, J. (2001). Meta-Analysis of Theory-of-Mind Development: The Truth about False Belief. Child Development, 72(3), 655-684

Whiten, A. (2005). Encyclopedia of Cognitive Science. Scotland: University of St. Andrews. 